\title{
Retrospective Analysis of Patients Undergoing Arthroscopic Rotator Cuff Repair at a Single Institution Yields a $0.11 \%$ Postoperative Infection Rate
}

\author{
Harsh Singh, B.A., Inis Isak, B.S., William M. Cregar, M.D., John D. Higgins, M.D., \\ Amar S. Vadhera, B.S., Allison K. Perry, B.S., Gregory P. Nicholson, M.D., \\ Brian J. Cole, M.D., M.B.A., and Nikhil N. Verma, M.D.
}

\begin{abstract}
Purpose: To establish an infection rate following primary arthroscopic rotator cuff repair (ARCR) from a single institutional database and to ascertain whether there is a relationship between the use of preoperative corticosteroid injection (CSI) and the risk of postoperative infection. Methods: All medical records at a single institution were retrospectively reviewed to identify patients who had undergone arthroscopic repair from January 2016 to December 2018. Patient charts were reviewed for CSI treatment within 6 months of surgery, superficial or deep infection within 2 months postoperatively, and specific treatment of the infection. Patient characteristics were summarized by descriptive statistics using means with standard deviations for continuous variables and frequencies with percentages for categorical variables. A $\chi^{2}$ correlation analysis was performed to determine the association between receiving an injection and having an infection. Results: A total of 1773 patients were included for analysis with an average age of $59.24 \pm 9.4$ years. The overall infection rate was $0.11 \%(2 / 1773$ patients). Both patients were treated with oral antibiotics. Of the included patients, 616 had a preoperative CSI within 6 months of their surgery, and 102 injections were administered within 1 month of surgery. None of these patients had a postoperative infection. A $\chi^{2}$ correlation analysis showed a negligible relationship between preoperative injections and postoperative infection $\left(\varphi=0.02, \chi^{2}=0.84\right)$. Conclusions: Through this single-institution, large cohort retrospective review, we found an overall $0.11 \%$ rate of postoperative infection following primary arthroscopic RCR. In addition, we found no correlation between the use of preoperative CSI ahead of elective ARCR at any time point and risk of developing a postoperative infection. Infection is uncommon following ARCR, and preoperative steroid injection did not increase infection risk in our study population. Level of Evidence: Level IV, therapeutic case series.
\end{abstract}

$\mathbf{R}$ otator cuff tears are estimated to affect $20.7 \%$ of the population, with the prevalence increasing with age. ${ }^{1}$ Initially, symptomatic tears are managed

From the Division of Sports Medicine, Department of Orthopedic Surgery, Rush University Medical Center, Chicago, Illinois, U.S.A.

The authors report the following potential conflicts of interest or sources of funding: G.P.N. reports other from the American Shoulder and Elbow Surgeons, other from Arthrosurface, other from Innomed, other from Tournier, and other from Wright Medical Technologies, outside the submitted work. B.J.C. reports other from Aesculap; other from National Institutes of Health; other from Operative Techniques in Sports Medicine; personal fees from Ossio; personal fees and other from Regentis; other from Smith $\theta$ Nephew; grants, personal fees, and other from Arthrex; other from Elsevier publishing; other from Bandgrip; other from Acumed LLC; other from Encore Medical; $L P$, other from GE Healthcare; other from Merck Sharp $\theta$ Dohme Corporation; other from SportsTek Medical; and other from Vericel, outside the submitted work. N.N.V. reports other from the American Orthopaedic Society for Sports Medicine, other from American Shoulder and Elbow Surgeons, other from Arthrex, other from Arthroscopy, other from Arthroscopy Association of with conservative modalities. Surgical intervention, most commonly in the form of rotator cuff repair (RCR), is indicated after nonresponse to nonoperative

North America, other from Breg, other from Cymedica, other from Knee, other from Minivasive, other from Omeros, other from Orthospace, other from Ossur, other from SLACK Incorporated, other from Smith $\theta$ Nephew, other from Vindico Medical-Orthopedics Hyperguide, and other from Wright Medical Technology, outside the submitted work; Full ICMJE author disclosure forms are available for this article online, as supplementary material.

Received April 6, 2021; accepted August 23, 2021.

Address correspondence to Nikhil N. Verma, M.D., Midwest Orthopaedics at Rush, 1611 W Harrison St., Chicago, IL 60661.E-mail: Nikhil.verma@ rushortho.com

(C) 2021 THE AUTHORS. Published by Elsevier Inc. on behalf of the Arthroscopy Association of North America. This is an open access article under the CC BY-NC-ND license (http://creativecommons.org/licenses/by-nc-nd/4.0/). 2666-061X/21490

https://doi.org/10.1016/j.asmr.2021.08.014 
treatment. ${ }^{2}$ Historically, RCR was performed in an open fashion; however, with advancement in technology, arthroscopic repair has become the standard of care. Over the last 25 years, the frequency of arthroscopic rotator cuff repair (ARCR) surgery in the United States has increased by $600 \%$. $^{3}$

The most common complications following RCR include arthrofibrosis, rotator cuff retear, and infection. ${ }^{4}$ Multiple risk factors have been linked to the increased risk of infection after RCR, including corticosteroid injections (CSIs). ${ }^{5}$ A systematic review from 2020 looked into the influence of CSIs before primary RCR on the risk of revision surgery, retears, and infections. ${ }^{6}$ Despite finding evidence supporting an increased risk of revision RCR, the authors could not find conclusive data to suggest an increased risk of postoperative infections following preoperative CSI use. ${ }^{6}$ As such, evidence needed to determine the influence of CSI on infection rates following ARCR is lacking. Further, recent studies using larger Medicare or private payor databases have reported infection rates between $1 \%$ and $2 \% .^{7,8}$ These reported rates were much greater than our internal experience and may reflect limitations in large database studies, prompting us to formally review our experience.

The purposes of our study were to establish an infection rate following primary ARCR from a single institutional database and to ascertain whether there is a relationship between preoperative CSI use and the risk of postoperative infection. We hypothesized that the overall rate of postoperative infection following primary ARCR would be low. In addition, we hypothesized that no significant association would exist between preoperative CSI use and postoperative infection rate.

\section{Methods}

After approval from an institutional review board, all medical records at a single institution were retrospectively reviewed to identify patients who had undergone arthroscopic repair from January 2016 to December 2018. The patient records were identified by using Current Procedural Terminology codes, including 29827 (arthroscopic rotator cuff repair). After we obtained a list of all these patients undergoing ARCR, an in-depth chart review was performed. The criterion for inclusion in the analysis was primary ARCR. Exclusion criteria were (1) revision RCR; (2) previous or current shoulder infection; (3) inadequate documentation to confirm the initial surgery or the subsequent follow-up appointments; and (4) concurrent use of immunosuppressive therapy. These ARCRs were performed by 1 of 6 board-certified sports medicine surgeons at our institution.

Patient charts were reviewed for CSI treatment within 6 months of surgery at the current or an outside facility, superficial or deep infection within 90 days postoperatively, emergency department or urgent care visits, and specific treatment of the infection. Superficial infections were defined as infections surrounding portals that resolved with antibiotics and did not require surgical intervention. ${ }^{9}$ Deep or complex infections were those that involved the deep subacromial space and/or required surgical debridement. Superficial and deep infections were delineated based on magnetic resonance imaging reviewed by the treating surgeon. For patients receiving subacromial injection for attempted nonoperative care of symptomatic rotator cuff tears, $80 \mathrm{mg}$ of methylprednisolone acetate injections was the standard of care for CSIs at our institution. Standard of care for perioperative (within 1 hour of incision) antibiotic prophylaxis included weight-based cefazolin or clindamycin if the patient was allergic to penicillin.

All patients underwent arthroscopic repair using suture anchor fixation. Procedures were performed at an ambulatory surgery center or hospital outpatient setting. Our standard wound care protocol includes dressing for 3 days, followed by dressing removal, and simple bandages over each portal site changed daily after a shower. Sutures are removed between 7 and 14 days after surgery.

\section{Statistical Analysis}

A statistical power analysis was performed to identify the patient sample size needed to find a $1 \%$ infection rate. Using a previously reported infection rate of $0.44 \%,{ }^{8} 1453$ patients were needed in the analysis to find a $1 \%$ infection rate. All statistical analyses were performed with Stata, version 16.1 (StataCorp., College Station, TX). Patient characteristics were summarized by descriptive statistics using means with standard deviations for continuous variables and frequencies with percentages for categorical variables. A $\chi^{2}$ correlation analysis was performed to determine the association between receiving an injection and having an infection. With one degree of freedom, a phi coefficient $(\varphi)$ was calculated, and statistical significance was defined when the $\chi^{2}>3.841$.

\section{Results}

A total of 1793 patients had ARCR surgery between 2016 and 2018. In total, 20 patients were excluded from our analysis for the following reasons: inadequate documentation to confirm postoperative follow-up $(\mathrm{n}=$ 11), revision rotator cuff surgery $(n=6)$, and concurrent use of immunosuppressive therapy $(\mathrm{n}=3)$. In total, 1773 (1134 male, 639 female) patients who underwent ARCR were included for final statistical evaluation. Average age of the included patients was $59.24 \pm 9.4$ years. The overall infection rate was $0.11 \%(2 / 1773$ patients). Both patients were diagnosed with a superficial infection surrounding the portals that resolved with a short course of oral Clindamycin. The first patient presented 1 month after their surgery with delayed 
wound healing, minimal serosanguinous discharge, and an area of warmth and redness around the incisions. The second patient presented 14 days after surgery with pain and minimal serosanguinous drainage from the lateral aspect of all incisions used. Both patients responded well with oral antibiotic therapy, with noted complete resolution of their symptoms.

Of the included patients, 616 had a preoperative CSI within 6 months of their surgery. In total, 378 of these injections were administered in the subacromial space, 164 in the glenohumeral joint, and 74 in the bicipital sheath. In total, 102 injections were administered within 1 month preoperatively and 326 between 1 and 3 months preoperatively. None of these patients had a postoperative infection. A $\chi^{2}$ correlation analysis showed a negligible relationship between preoperative injections and postoperative infection $\left(\varphi=0.02, \chi^{2}=0.84\right)$.

\section{Discussion}

At our institution, infection after arthroscopic RCR surgery is rare. Our reported infection rate of $0.11 \%$ following primary ARCR is similar to previous reports in the literature. ${ }^{4,9,10}$ Brislin et al. $^{4}$ retrospectively reviewed a small single institution cohort following ARCR over a 6-month period looking at 90-day complications. They included a total of 263 patients and reported an all-complication rate of $10.6 \%$ with 1 deep postoperative infection reported, lending to a $0.38 \%$ infection rate. ${ }^{4}$ This required formal operative debridement and antibiotic therapy. ${ }^{4}$ Randelli et al. ${ }^{11}$ completed a large survey-based study involving 59 surgeons and 9385 arthroscopic shoulder surgeries. They reported an overall infection rate of $0.12 \%$ following ARCR. ${ }^{11}$ They further analyzed their results based on the use of antibiotic prophylaxis and reported a lower infection rate of $0.095 \%$ when perioperative antibiotic prophylaxis was used. ${ }^{11}$ Herrera et al. ${ }^{12}$ reported a $1.9 \%$ postoperative infection rate following arthroscopically assisted mini-open RCR over a 9-year period (7/360) across 2 institutions. They performed a subacromial decompression arthroscopically followed by a mini-open RCR. ${ }^{12}$ When performing a second surgical scrub and separate draping before the miniopen procedure, they reported no infections in more than 200 consecutive cases. ${ }^{12}$ These previous studies either involve a small cohort, are multi-institutional, or encompass open RCR in their analysis. ${ }^{10-12}$ Through this study, we establish a postoperative infection risk of $0.11 \%$ involving a large cohort of consecutive patients over a 2-year period at a single institution.

We found no significant correlation between preoperative CSI at any time point or any location within the shoulder and subsequent postoperative infection following primary ARCR. Both patients who sustained a superficial infection postoperatively did not undergo a preoperative injection. Previous literature has evaluated the effects of preoperative CSI on RCR surgery. ${ }^{5,13}$ Notably, Forsythe et al. ${ }^{5}$ performed a large retrospective study using a private payor database involving more than 60,000 patients and stratified their analysis based on timing of preoperative CSI ahead of arthroscopic RCR surgery. ${ }^{5}$ They found a significantly greater rate of postoperative surgical-site infection (SSI) in patients who received a CSI within 1 month of surgery (1.3\%) versus those that did not $(0.8 \%))^{5}$ Further, through multivariate analysis, they concluded that preoperative CSI within 1 month of surgery is an independent risk factor for postoperative SSI. ${ }^{5}$ Similarly, Werner et al. ${ }^{13}$ also performed a large-scale retrospective study using the Medicare database to report an increase in postoperative SSI when ipsilateral intra-articular CSIs were administered within 3 months of arthroscopic shoulder surgery They included patients undergoing allarthroscopic shoulder surgery rather than isolating just arthroscopic RCR. ${ }^{13}$ There are several important limitations to consider with these large-scale database studies that could explain differences in findings from our study. First, results hinge on accuracy of coding across multiple institutions. Second, specifics of injections including corticosteroid mixtures, injection location, and techniques can differ drastically across institutions. Lastly, patient comorbidities, surgical indications, and surgeon skill levels can vary across institutions. Single-institution studies, such as our study, have the benefit of mitigating many of these aforementioned variables that can skew effects of interventions. In addition, we include a large cohort of patients in our analysis, which can often be a limitation of single-institution studies. We are unable to explain the large disparity in infection rate between the institutional studies and our current experience. In our opinion, the differences reflect the difficulty in abstracting complex data from databases that rely on accurate physician International Classification of Diseases, Ninth Revision, or Tenth Revision coding. Improper coding likely overestimates the incidence of infection. In addition, these databases are unlikely to capture superficial infections that are treated without further surgery. Another reason for the discrepancy may be that a large portion of these infections are seen in the emergency department setting or via a phone call, and, therefore, not by an orthopaedic surgeon. Therefore, to achieve an accurate representation of clinical experience, using actual clinical data is best, but of course achieving adequate sample size is challenging. Because of the nature of our institution, and the numbers of procedures performed by a large number of sports medicine and shoulder fellowship-trained surgeons, we are uniquely able to report these results.

\section{Limitations}

There are several limitations to note when interpreting the results of this study. First, this is a retrospective 
chart review study, which could introduce bias. Second, we did not include patient demographic variables such as diabetes, obesity, or smoking status or the American Society of Anesthesiologists' class and surgical time, which may be potential confounders and, therefore, influence infection rate. However, given the small number of infections identified in this study, it would have been impossible to draw any meaningful conclusions regarding infection risk and preoperative risk factors. In addition, it is possible some patients who received an injection at an outside facility were missed during chart review. Third, given the rate of infection is extremely low $(0.11 \%)$, increasing the cohort size could help better determine the correlation between preoperative CSI and development of a postoperative infection. Lastly, it is possible that some "indolent" shoulder infections may have been missed by the treating surgeon or were addressed in the emergency department or urgent care setting. Low-grade infections have been suggested as a potential cause of persistent shoulder pain, stiffness, or failure of healing following rotator cuff surgery and are controversial and difficult to diagnose. Therefore, it is possible that these infections existed within our patient population but were unrecognized.

\section{Conclusions}

Through this single-institution, large cohort retrospective review, we found an overall $0.11 \%$ rate of postoperative infection following primary arthroscopic RCR. In addition, we found no correlation between the use of preoperative CSI ahead of elective ARCR at any time point and risk of developing a postoperative infection. Infection is uncommon following ARCR, and preoperative steroid injection at our institution did not increase infection risk in our study population.

\section{References}

1. Yamamoto A, Takagishi K, Osawa T, et al. Prevalence and risk factors of a rotator cuff tear in the general population. J Shoulder Elbow Surg 2010;19:116-120.

2. Ryösä A, Laimi K, Äärimaa V, Lehtimäki K, Kukkonen J, Saltychev M. Surgery or conservative treatment for rotator cuff tear: A meta-analysis. Disabil Rehabil 2017;39: 1357-1363.

3. Colvin AC, Egorova N, Harrison AK, Moskowitz A, Flatow EL. National trends in rotator cuff repair. J Bone Joint Surg Am 2012;94:227-233.

4. Brislin KJ, Field LD, Savoie FH 3rd. Complications after arthroscopic rotator cuff repair. Arthroscopy 2007;23: 124- 128.

5. Forsythe B, Agarwalla A, Puzzitiello RN, Sumner S, Romeo AA, Mascarenhas R. The timing of injections prior to arthroscopic rotator cuff repair impacts the risk of surgical site infection. J Bone Joint Surg Am 2019;101: 682-687.

6. Kunze KN, Mirzayan R, Beletsky A, et al. Do corticosteroid injections before or after primary rotator cuff repair influence the incidence of adverse events? A subjective synthesis. Arthroscopy 2020;36:1476-1484.

7. Jensen AR, Cha PS, Devana SK, et al. Evaluation of the trends, concomitant procedures, and complications with open and arthroscopic rotator cuff repairs in the Medicare population. Orthop J Sports Med 2017;5:23259671 17731310.

8. Cancienne JM, Brockmeier SF, Carson EW, Werner BC. Risk factors for infection after shoulder arthroscopy in a large Medicare population. Am J Sports Med 2018;46: 809-814.

9. Peel AL, Taylor EW. Proposed definitions for the audit of postoperative infection: A discussion paper. Surgical Infection Study Group. Ann R Coll Surg Engl 1991;73: 385-388.

10. Hughes JD, Hughes JL, Bartley JH, Hamilton WP, Brennan KL. Infection rates in arthroscopic versus open rotator cuff repair. Orthop J Sports Med 2017;5: 2325967117715416.

11. Randelli P, Castagna A, Cabitza F, Cabitza P, Arrigoni P, Denti M. Infectious and thromboembolic complications of arthroscopic shoulder surgery [published correction appears in J Shoulder Elbow Surg. 2014;23:598]. J Shoulder Elbow Surg 2010;19:97-101.

12. Herrera MF, Bauer G, Reynolds F, Wilk RM, Bigliani LU, Levine WN. Infection after mini-open rotator cuff repair. J Shoulder Elbow Surg 2002;1 1:605-608.

13. Werner BC, Cancienne JM, Burrus MT, Griffin JW, Gwathmey FW, Brockmeier SF. The timing of elective shoulder surgery after shoulder injection affects postoperative infection risk in Medicare patients. J Shoulder Elbow Surg 2016;25:390-397. 